\title{
COMPETÊNCIAS DA ORGANIZAÇÃO INOVADORA: UM ESTUDO EM EMPRESAS DO INSTITUTO PAULISTA DE EXCELÊNCIA DA GESTÃO E DA FUNDAÇÃO NACIONAL DA QUALIDADE
}

\author{
COMPETENCIES OF THE INNOVATIVE ORGANIZATION: A STUDY OF BUSINESSES OF THE INSTITUTO \\ PAULISTA DE EXCELÊNCIA DA GESTÃO AND FUNDAÇÃO NACIONAL DA QUALIDADE
}

\begin{abstract}
LAS COMPETENCIAS DE LA ORGANIZACIÓN INNOVADORA: UN ESTUDIO EN EMPRESAS DEL INSTITUTO PAULISTA DE EXCELENCIA DE LA GESTIÓN Y DE LA FUNDACIÓN NACIONAL DE LA CALIDAD
\end{abstract}

\author{
ADRIANA BARALDI \\ Doutoranda \\ Escola Superior de Propaganda e Marketing (ESPM), Brasil \\ adrianabaraldi@yahoo.com.br \\ MARCOS AUGUSTO DE VASCONCELLOS \\ Doutor \\ Fundação Getúlio Vargas (FGVIEAESP), Brasil \\ marcosav@terra.com.br \\ LUIZ CARLOS DI SERIO \\ Doutor \\ Fundação Getúlio Vargas (FGV/EAESP), Brasil \\ luiz.diserio@fgv.br \\ SILVANA MARQUES DOS SANTOS PEREIRA \\ Doutor \\ Fundação Getúlio Vargas (FGV/EAESP), Brasil \\ silvana.pereira@fgv.br \\ ALEXANDRE LUIS PRIM \\ Doutorando \\ Fundação Getúlio Vargas (FGV/EAESP), Brasil \\ alexandrelprim@gmail.com \\ Submetido em: 29/12/2015 \\ Aprovado em: 22/08/2016
}

Doi: alcance.v23n3.p312-328

\section{RESUMO}

Gerir a inovação tem sido o desafio de boa parte das organizações, uma vez que demanda competências específicas às empresas que quando bem geridas podem significar ganho competitivo, mas quando não, podem representar estagnação e perda de mercado. Com base nisso, o artigo tem como objetivo identificar lacunas na gestão de inovação de empresas do Instituto Paulista de Excelência da Gestão e da Fundação Nacional da Qualidade com base na metodologia do Fórum de Inovação da FGVIEAESP, que tem como base cinco competências da organização inovadora: liderança e intenção estratégica; meio inovador interno; pessoas; processos de inovação; e resultados. Para tanto, foi realizado um estudo quantitativo. 0 método de pesquisa adotado foi a survey. Como análise estatística foi realizada análise fatorial confirmatória, purificação dos dados, de acordo com os indicadores de confiabilidade, análise do modelo estrutural e análise de regressão. Foi enviado 
questionário on-line para todas as empresas do Instituto Paulista de Excelência da Gestão e da Fundação Nacional da Qualidade e obtido um total de 93 respostas válidas. Os resultados demonstraram que as competências "meio inovador", "pessoas" e "processos" são responsáveis pelo desempenho obtido na competência "resultado". Apenas a "liderança" não apareceu como determinante do resultado. Como contribuição, o artigo chama a atenção para a dificuldade de as organizações gerarem resultado de inovação e o coloca como consequência de competências que 0 antecedem.

Palavras-chave: Inovação. Gestão da Inovação. Organização Inovadora.

\begin{abstract}
Managing innovation has been a challenge for most organizations, as it requires specific skills of companies that when well managed, can result in gains in competitiveness, but when not well managed, can lead to stagnation and loss of market share. In this context, this paper aims to identify gaps in the management of innovation of companies of the Instituto Paulista de Excelência da Gestão and Fundação Nacional da Qualidade [Sao Paulo Institute of Management Excellence and National Quality Foundation], based on the methodology of the Innovation Forum of the FGV/EAESP, which is based on five competencies of the innovative organization: leadership and strategic intent; internal innovative environment; people; processes of innovation; and results. For this purpose, a quantitative study was carried out. A survey was adopted as our research method. We then performed confirmatory factor analysis, data purification, according to the reliability indicators, analysis of the structural model, and regression analysis. An online questionnaire was sent to all the companies of the Instituto Paulista de Excelência da Gestão and Fundação Nacional da Qualidade, and 93 valid responses were received. The results showed that the competencies "innovative environment", "people" and "processes" are responsible for the performance obtained in the "results" competence. Only "leadership" competence did not appear as a determinant of the results. As a contribution, the paper draws attention to the difficulties faced by organizations in creating the result of innovation, placing it as a consequence of the competencies that preceded it.
\end{abstract}

Keywords: Innovation. Management of Innovation. Innovative Organization.

\title{
RESUMEN
}

Administrar la innovación ha sido el desafío de una buena parte de las organizaciones, ya que demanda de las empresas competencias específicas que, cuando están bien administradas, pueden significar ventajas competitivas, pero cuando no lo están, pueden representar estancamiento y pérdida de mercado. Con base en ello, el artículo tiene como objetivo identificar vacíos en la gestión de innovación de empresas del Instituto Paulista de Excelencia de la Gestión y de la Fundación Nacional de la Calidad con base en la metodología del Fórum de Innovación de la FGV/EAESP que tiene como base cinco competencias de la organización innovadora: liderazgo e intención estratégica; medio innovador interno; personas; procesos de innovación; y resultados. Para eso fue realizado un estudio cuantitativo. El método de investigación adoptado fue la survey. Como análisis estadístico se hizo un análisis factorial confirmatorio, depuración de los datos de acuerdo con los indicadores de confiabilidad, análisis del modelo estructural y análisis de regresión. El cuestionario on-line fue enviado a todas las empresas del Instituto Paulista de Excelencia de la Gestión y de la Fundación Nacional de la Calidad, y se obtuvieron un total de 93 respuestas válidas. Los resultados demostraron que las competencias "medio innovador", "personas" y "procesos" son responsables por el desempeño obtenido en la competencia "resultado". Solamente el "liderazgo" no apareció como determinante del resultado. Como contribución, el artículo llama la atención hacia la dificultad de las organizaciones para generar un resultado de innovación y lo coloca como consecuencia de competencias que lo anteceden.

Palabras clave: Innovación. Gestión de la Innovación. Organización Innovadora.

\section{INTRODUÇÃO}

Organizações inovadoras inovam sistematicamente e conseguem transformar competências em resultados de inovação (BARBIERI, 2003; CROSSAN; APAYDIN, 2009; HARGADON; SUTTON, 2000; SMITH et al., 2008). Embora a inovação tenha o potencial de conferir às organizações ganhos competitivos sustentáveis em 
longo prazo (CARAYANNIS; GRIGOROUDIS, 2014; CARAYANNIS; SAMARA; BAKOUROS, 2015; HEGER; ROHRBECK, 2012), gerar resultados de inovação tem sido um desafio (QUANDT et al., 2014; RAMOS; ZILBER, 2015).

Frente a esse cenário, não basta apenas inovar, as organizações devem ser capazes de inovar de forma sistemática (CROSSAN; APAYDIN, 2009; HARGADON; SUTTON, 2000) e encontrar alternativas de inovação que melhor representem o negócio (MONTEIRO, 2015; PONOMARIOV; TOIVANEN, 2014). Entretanto, apesar da literatura de inovação preconizar que as organizações podem se tornar competitivas com a inovação e que estas devem gerir a inovação para se tornarem organizações inovadoras, ela não é específica em como isso pode ser feito, havendo a necessidade identificar os fatores que possibilitam uma boa gestão da inovação e considerá-los como holísticos e interligados (SMITH et al., 2008). E ao mesmo tempo, poucas foram as tentativas de reunir todo o conhecimento que se tem em inovação em um único modelo (CROSSAN; APAYDIN, 2009; SMITH et al., 2008).

Dentre os modelos existentes, pode-se destacar o proposto pelo Fórum de Inovação FGV/EAESP (2014), que apresenta uma visão integrada da capacidade de inovação da organização, sendo composto por cinco competências essenciais, sendo elas: liderança e intenção estratégica; meio inovador interno; pessoas; processos de inovação; e resultados. O modelo parte de uma visão holística e integrada da inovação e considera tanto os fatores internos e externos à organização como integrantes do processo de inovação, além de considerar que toda organização precisa das mesmas competências para se tornar e se manter inovadora, pois o que pode variar é a operacionalização dos conceitos, conforme as características específicas de cada organização (Fórum de Inovação FGVIEAESP, 2014). O modelo consiste, portanto, numa tentativa de prover subsídios para que as organizações possam orientar suas práticas de inovação em prol de melhores resultados de inovação e na busca por melhor desempenho e vantagens competitivas duradouras.

Dada essas considerações, o artigo tem como objetivo identificar lacunas na gestão de inovação de empresas do Instituto Paulista de Excelência da Gestão e da Fundação Nacional da Qualidade com base na metodologia do Fórum de Inovação da FGV/EAESP. E como questões norteadoras: como gerir a inovação? e quais as competências diretamente relacionadas a geração da inovação? $O$ artigo está dividido em seis sessões: introdução; referencial teórico; procedimentos metodológicos; apresentação e discussão dos resultados; e considerações finais, limitações e recomendações.

\section{REFERENCIAL TEÓRICO}

\subsection{Gestão da inovação}

A gestão da inovação está relacionada com a criação de condições para que a inovação ocorra de forma contínua e permanente (TIDD; BESSANT; PAVITT, 2008). Organizações mais inovadoras possuem, portanto, melhores condições para desenvolverem inovações, enquanto organizações menos inovadoras possuem um ambiente menos propício ao seu desenvolvimento (Fórum de Inovação FGV/EAESP, 2014).

Para se tornarem inovadoras e criarem, assim, um ambiente propício ao desenvolvimento de inovações, as organizações devem evoluir de competências estáticas para dinâmicas e se readequarem constantemente em meio às rápidas mudanças no ambiente (WEERAWARDENA; MAVONDO, 2011). Para isso, as práticas organizacionais devem ser pensadas e desenvolvidas de forma integrada e contínua, no intuito de identificar e explorar oportunidades que permitam obter resultados maximizados e visão de negócio (CHESBROUGH, 2010).

A inovação requer, portanto, um conjunto de atividades que englobem criatividade, criação e entrega de produtos/serviços/processos, gestão de recursos, oportunidades de reconhecimento e estratégias para clientes e que possibilitem práticas coordenadas de criação, avaliação, aceitação, adoção, difusão e comercialização de uma ideia (ALSAATY, 2011). Tais aspectos podem ser conquistados mediante a formação de competências de inovação, conforme discutido na próxima seção.

\subsection{Competências da organização inovadora}

A organização inovadora pode ser definida como aquela em que as pessoas estão envolvidas (CROSSAN; APAYDIN, 2009; MACHADO, 2007), ou, em termos quantitativos, em que mais da metade de seus colaboradores está engajada nos processos de inovação. Estas organizações se destacam por se antecipar aos seus concorrentes e identificar oportunidades ainda não exploradas, além de conseguir ditar as regras do jogo em seu ambiente de competição (MARKIDES, 1997). O Fórum de Inovação FGVIEAESP (2014), cujo modelo de

Revista Alcance - Eletrônica - vol. 23 - n. 3 - jul./set. 2016 
competências da organização inovadora serve como guia para este estudo, define a organização inovadora como a que pratica a inovação sistemática, sendo esta permeada por um processo contínuo e permanente de produção de inovações, de qualquer natureza - produto, processo, gestão ou negócio - e de qualquer magnitude.

\section{Figura 1: Modelo de Diagnóstico da Organização Inovadora}

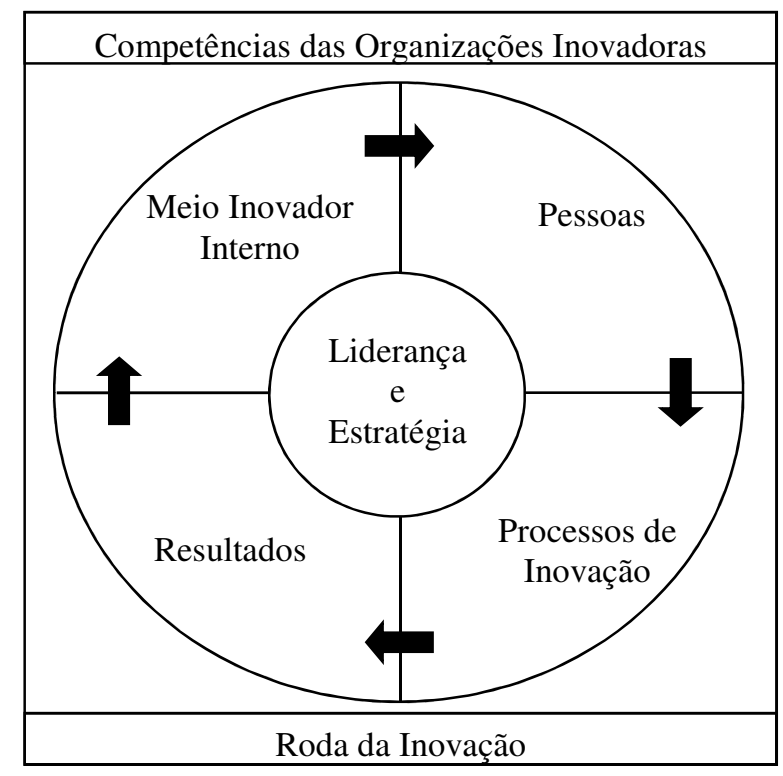

Fonte: Fórum de Inovação FGV/EAESP (2014).

A organização inovadora pode ser identificada mediante a análise das competências essenciais que uma organização deve possuir para ser inovadora, as quais representam as condições necessárias para que a inovação ocorra (HARGADON; SUTTON, 2000; MACHADO, 2007). Uma competência essencial é definida como um conjunto de fatores que contribuem para a prosperidade ou sucesso em longo prazo de uma organização (CHRISTENSEN, 1997; HAMEL; PRAHALAD, 1994). Considerando a definição de organização inovadora, podese definir competência inovadora como um conjunto de fatores que contribuem para que uma organização torne e mantenha com sucesso uma organização inovadora. O Fórum de Inovação FGV/EAESP (2014) traz como proposta de competências inovadoras: liderança e intenção estratégica; meio inovador interno; pessoas; processos de inovação; e resultados. Estas competências formam o modelo de diagnóstico da organização inovadora, chamada de Roda da Inovação (Figura 1), que representa uma visão dinâmica das organizações inovadoras.

\subsubsection{Liderança e intenção estratégica}

A liderança e a intenção estratégica definem os rumos e proporcionam um ambiente adequado à criatividade e à inovação (CROSSAN; APAYDIN, 2009; TANG, 1998). Esta competência tem duas funções básicas e complementares: a de direção, que permite a definição de rumos e a de intercâmbios com o meio, que permite à organização captar os elementos externos que incidem sobre ela (Fórum de Inovação FGV/EAESP, 2014).

A direção é composta pelos princípios corporativos, que orientam as tomadas de decisão e a natureza das relações entre as pessoas (CROSSAN; APAYDIN, 2009; MACHADO, 2007; SMITH et al., 2008) e pela intenção estratégica para inovação, que define em que medida a inovação está inserida na estratégia organizacional e corresponde à visão que a organização tem sobre si mesma em termos de inovação (CROSSAN; APAYDIN, 2009; TANG, 1998). Intercâmbios com o meio inclui a ação institucional, que compreende um conjunto de ações da organização que podem ajudar a criar um clima favorável às inovações na empresa (BARBIERl; ÁLVARES, 2002); e prospecção de sinais, corresponde à capacidade de traduzir os dados do ambiente em informações adequadas para tomadas de decisão inteligentes, identificando tanto as necessidades de inovação para enfrentar ameaças e solucionar fraquezas internas, como as oportunidades de inovação (OLIVEIRA; ALVES, 2014). 

resultado.

H1. A competência liderança e intenção estratégica influencia positivamente a competência

\subsubsection{Meio inovador interno}

A expressão meio inovador foi criada inicialmente para designar um local ou região em que está instalado um conjunto de organizações que atuam de forma sinérgica para gerar continuamente inovações ou combinações de inovações (CASTELLS; HALL, 1994). Por analogia, Barbieri e Álvares (2002) cunharam a expressão meio inovador interno, adotada pelo Fórum de Inovação FGV/EAESP, para designar o contexto que propicia as sinergias internas necessárias à geração continuada de inovações em uma dada organização. Os fatores determinantes do meio inovador interno são a liderança mobilizadora, as relações formais e as relações socioculturais (Fórum de Inovação FGV/EAESP, 2014).

A liderança mobilizadora é a que estimula o engajamento e a mobilização de todos em prol da inovação (CROSSAN; APAYDIN, 2009; HURLEY; HULT, 1998; LAWSON; SAMSON; SIMÕES, 2001). Pode-se dizer que a liderança mobilizadora consiste no compartilhamento de poder e na concessão de autonomia para a tomada de decisões (BENNIS; NANUS, 1988). Já as relações formais são as derivadas do organograma e dos instrumentos de administração, como manuais, normas e regulamentos, que constituem o modelo de gestão da organização e determinam a qualidade de vida no trabalho (SPISAK et al., 2015). No modelo de gestão é importante que a organização tenha um projeto de trabalho motivador e que estimule a inovação (ZACHER; ROSING, 2015). As relações socioculturais compreendem interações e relacionamentos informais, que se estabelecem espontaneamente entre pessoas e grupos e moldam a cultura de inovação na organização (SMITH et al., 2008; ZACHER; ROSING, 2015). A inovação deve, portanto, permear toda a organização, ter estrutura sistêmica e todos serem responsáveis por sua promoção e geração (ÁLVARES, 2001).

\section{H2. A competência meio inovador interno influencia positivamente a competência resultado.}

\subsubsection{Pessoas}

Esta competência trata de características especificas de cada pessoa e é essencial para cumprir com efetividade o seu papel de agente de inovação e mudanças (Fórum de Inovação FGV/EAESP, 2014). Tais aspectos são a qualificação e aprendizagem e a motivação. $O$ estímulo ao aprendizado contínuo - individual e coletivo - é uma característica marcante das organizações inovadoras (BAO; CHEN; ZHOU, 2012; SALUNKE; WEERAWARDENA; MCCOLL-KENNEDY; 2011). Além do estímulo, essas organizações buscam alinhar a capacitação dos colaboradores à intenção estratégica de inovação, além de prover infraestrutura e sistemas que facilitem o compartilhamento de conhecimento entre as pessoas (ZACHER; ROSING, 2015). Para tanto, a organização deve estimular a motivação intrínseca, resultante do trabalho em si, que depende de três fatores: significado percebido, grau em que a pessoa percebe o trabalho como importante, valioso e significativo; responsabilidade percebida, até que ponto 0 indivíduo se sente pessoalmente responsável pelos resultados do trabalho que executa; e conhecimento dos resultados, até onde o indivíduo recebe feedback e pode avaliar o quão efetivamente está executando o seu trabalho (Fórum de Inovação FGVIEAESP, 2014; VASCONCELLOS, 1997).

\section{H3. A competência pessoas influencia positivamente a competência resultado.}

\subsubsection{Processos de inovação}

Esta competência diz respeito à realização das inovações e inclui: gerenciamento dos processos de inovação, obtenção dos recursos para inovação e etapas dos processos de inovação (Fórum de Inovação FGV/EAESP, 2014). 0 gerenciamento dos processos de inovação deve consistir num processo contínuo (TIDD; BESSANT; PAVITT, 2008), pois representam os comandos operacionais das atividades necessárias à geração de inovações (JOHNSON; CHRISTENSEN; KAGERMANN, 2008), sendo eles: planejamento das inovações estratégicas; monitoramento dos processos de inovação; avaliação das competências inovadoras e dos resultados das inovações; feedback sobre os resultados e impactos das inovações; análise dos projetos de inovação e melhoria, tanto os bem como os mal sucedidos, em busca de lições para inovações futuras; e comemoração das inovações bem-sucedidas (BARBIERI, 2003). 
Os recursos para inovação constituem um fator crítico para as organizações, pois determinam o que a empresa pode realizar em termos de inovação (CHRISTENSEN; OVERDORF, 2000). Os recursos básicos para a inovação são o capital e o conhecimento (SILVA; REIS, 2015). As organizações inovadoras dispõem de métodos rotineiros para mapear as fontes de financiamento e para avaliar os riscos das inovações (BORCHARDT; SANTOS, 2014). Sobre o conhecimento para inovação, este é a matéria-prima fundamental dos processos de inovação e a chave para a produção contínua de inovações (BAO; CHEN; ZHOU, 2012; CHESBROUGH, 2012; SALUNKE; WEERAWARDENA; MCCOLL-KENNEDY, 2011). Organizações inovadoras têm tido sucesso devido à sua habilidade e expertise na criação de conhecimento organizacional, o que significa a capacidade de uma empresa, como um todo, criar novos conhecimentos, disseminá-los e incorporá-los por toda a organização (CROSSAN; APAYDIN, 2009). O conhecimento também pode ser adquirido de fora da organização, mediante alianças estratégicas (FLETCHER; HARRIS, 2012).

\section{H4. A competência processos de inovação influencia positivamente a competência resultado.}

\subsubsection{Resultados}

A geração de resultados de inovação consiste em um desafio para as organizações (QUANDT et al., 2014; RAMOS; ZILBER, 2015). Os resultados podem ser analisados sob três prismas complementares: resultados imediatos das inovações, impactos das inovações e cumprimento dos objetivos e planos de inovação (Fórum de Inovação FGV/EAESP, 2014).

Os resultados imediatos das inovações trazem como perspectiva avaliar o sucesso (ou não) de cada inovação (BARBIERI, 2003). Os impactos das inovações são as consequências (planejadas ou não) das inovações (ROBERTSON; CASALI; JACOBSON, 2012). Do ponto de vista do modelo de diagnóstico da organização inovadora do Fórum de Inovação FGV/EAESP (2014), um projeto de mudança somente será considerado uma efetiva inovação se apresentar resultados que: sejam positivos para todos os stakeholders, sem impactos negativos de qualquer espécie; e não sejam efêmeros, isto é, que apresentem benefícios por algum período de tempo, até que a inovação seja aperfeiçoada ou substituída por outra.

Por fim, o cumprimento dos objetivos e planos de inovação consiste em avaliar se foram cumpridos os planos de inovação estabelecidos no gerenciamento dos processos de inovação, o que permite alinhar o que está sendo planejado em termos de inovação com as ações e resultados conquistados (TIDD; BESSANT; PAVITT, 2008).

\section{H5. A competência resultado é influenciada positivamente pelas competências liderança e intenção estratégica; meio inovador interno; pessoas e processos de inovação.}

\section{PROCEDIMENTOS METODOLÓGICOS}

O estudo teve como objetivo identificar lacunas na gestão de inovação de empresas mantenedoras do Instituto Paulista de Excelência da Gestão e da Fundação Nacional da Qualidade, com base na metodologia do Fórum de Inovação FGV/EAESP. Para tanto, foi realizado um estudo quantitativo (SAUNDERS; LEWIS; THORNILL, 2007). O método de pesquisa adotado foi a survey (BABBIE, 2001), considerado adequada para os propósitos desta pesquisa por permitir alcançar um quantitativo maior de respondentes.

As empresas mantenedoras do Instituto Paulista de Excelência da Gestão e da Fundação Nacional da Qualidade foram escolhidas como objeto de estudo, sobretudo por buscarem desenvolver e melhorar a gestão das organizações a ela filiadas em todo o país. Estes órgãos reúnem empresas de todos os portes e setores, o que permite uma visão global do contexto de inovação das empresas brasileiras.

Como instrumento de pesquisa foi utilizado um questionário desenvolvido com base na metodologia do Fórum de Inovação FGVIEAESP. As questões foram desenvolvidas em escala Likert de cinco pontos, variando entre 1 para Discordo Totalmente e 5 para Concordo Totalmente. Foi elaborado um total de 35 perguntas, distribuídas em: (a) 13 perguntas sobre o perfil da organização; (b) 04 perguntas sobre a competência Liderança e Intenção Estratégica; (c) 05 perguntas sobre a competência Meio Inovador Interno; (d) 02 perguntas sobre a competência Pessoas; (e) 07 perguntas sobre a competência Processos de Inovação; e (f) 04 perguntas sobre a competência Resultados. 0 Quadro 1 apresenta as competências e as dimensões que guiaram a pesquisa e a construção do questionário. 
Quadro 1: Categorias, Dimensões e Indicadores

\begin{tabular}{|c|c|c|}
\hline $\begin{array}{l}\text { Competências } \\
\text { Inovadoras }\end{array}$ & Dimensões de Inovação & Fatores de Inovação \\
\hline \multirow{2}{*}{$\begin{array}{l}\text { Liderança e Intenção } \\
\text { Estratégica }\end{array}$} & Direção & $\begin{array}{l}\text { - Princípios corporativos } \\
\text { • Intenção estratégica para a inovação }\end{array}$ \\
\hline & Intercâmbio com o meio & $\begin{array}{l}\text { - Ação institucional } \\
\text { • Prospecção e interpretação de sinais }\end{array}$ \\
\hline \multirow{3}{*}{ Meio Inovador Interno } & Liderança & - Liderança mobilizadora \\
\hline & Relações formais & $\begin{array}{l}\text { - Modelo de gestão } \\
\text { - Qualidade de vida do trabalho }\end{array}$ \\
\hline & Relações informais & $\begin{array}{l}\text { - Cultura e inovação } \\
\text { • Redes internas informais }\end{array}$ \\
\hline Pessoas & Pessoas & $\begin{array}{l}\text { - Qualificação e aprendizagem } \\
\text { • Motivação }\end{array}$ \\
\hline \multirow{3}{*}{ Processos de Inovação } & Gerenciamento das inovações & - Gestão dos processos de inovação \\
\hline & Recursos para inovação & $\begin{array}{l}\text { - Capital para inovação } \\
\text { - Conhecimento para inovação } \\
\text { - Alianças estratégicas para inovação }\end{array}$ \\
\hline & $\begin{array}{l}\text { Etapas dos processos das } \\
\text { inovações }\end{array}$ & $\begin{array}{l}\text { - Geração de ideias para inovação } \\
\text { - Priorização e desenvolvimento de projetos } \\
\text { - Implementação }\end{array}$ \\
\hline Resultados & Resultados das inovações & $\begin{array}{l}\text { - Resultados econômico-financeiros } \\
\text { - Cumprimento de objetivos de inovação } \\
\text { - Resultados do processo de inovação } \\
\text { - Impactos das inovações }\end{array}$ \\
\hline
\end{tabular}

Fonte: Fórum de Inovação FGV/EAESP (2014).

A partir do desenvolvimento do questionário a ser aplicado, as seguintes etapas foram cumpridas: (1) aquisição de licença SurveyMonkey para realização do levantamento e da análise de dados nessa plataforma; (2) elaboração do processo de comunicação e material de estímulo aos respondentes da pesquisa; (3) inclusão do questionário na plataforma SurveyMonkey; (4) definição do universo amostral a ser utilizado em etapa piloto da pesquisa (25 participantes do Núcleo Temático de Gestão da Inovação - FNQ); (5) teste piloto do questionário; e (6) feedback telefônico da etapa piloto.

Após o teste piloto, o questionário foi revisado e incluído na plataforma SurveyMonkey, e seu link foi disponibilizado numa newsletter (convite), tendo o Instituto Paulista de Excelência da Gestão e a Fundação Nacional da Qualidade como remetentes. Essa newsletter foi enviada para todas as empresas do Instituto Paulista de Excelência da Gestão e da Fundação Nacional da Qualidade por meio da plataforma MailChim. No intuito de reforçar a participação das empresas na pesquisa, foram realizadas 3 rodadas de ligações para todas as empresas, além de reenvio de newsletter aos não respondentes de acordo com avaliação semanal realizada junto ao banco de dados que identificava as empresas respondentes. Ao final, foram obtidos 93 questionários válidos. Finalmente, os dados coletados na plataforma SurveyMonkey foram exportados para o MicroSoft Excel. 
A análise estatística incluiu uma análise inicial para verificar se todos os questionários eram válidos. Assim, inicialmente foi obtido um total de 117 questionários, dos quais 93 continham todas as questões preenchidas, totalizando os questionários válidos utilizados nesta pesquisa. Foi feita uma análise descritiva e, posteriormente, uma análise fatorial confirmatória como forma de validar as categorias, as dimensões e os indicadores presentes no modelo de diagnóstico da organização inovadora proposto pelo Fórum de Inovação da FGV/EAESP. A análise fatorial consiste numa técnica de análise multivariada em que todas as variáveis são analisadas em conjunto, a fim de verificar as inter-relações existentes entre elas, tendo em vista identificar quais variáveis conseguem diferenciar grupos ou categorias a serem analisadas (HAIR et al., 1998), sendo, portanto, adequada aos propósitos dessa pesquisa. Em seguida, foi feita a purificação dos dados de acordo com os indicadores de confiabilidade no Quadro 2.

Quadro 2: Indicadores de confiabilidade da AFC

\begin{tabular}{|l|l|l|l|}
\hline Indicador & Descrição do indicador & Critério & Aporte teórico \\
\hline Carga padronizada & $\begin{array}{l}\text { Indica a convergência entre } \\
\text { construtos }\end{array}$ & $>0,70$ & Hair et al. (2009) \\
\hline $\mathrm{R}^{2}$ & $\begin{array}{l}\text { Representa a variação das } \\
\text { variáveis observadas } \\
\text { referentes a um construto }\end{array}$ & $>0,50$ & Hair et al. (2009) \\
\hline Alfa de Cronbach & $\begin{array}{l}\text { Mostra a consistência } \\
\text { interna do construto }\end{array}$ & $>0,70$ & Kline (2011) \\
\hline $\begin{array}{l}\text { Variância média extraída } \\
\text { (AVE - Average variance } \\
\text { extracted) }\end{array}$ & $\begin{array}{l}\text { Exibe quanta variância nos } \\
\text { indicadores é explicada pelo } \\
\text { construto }\end{array}$ & $>0,50$ & Hair et al. (2009) \\
\hline Confiabilidade composta & $\begin{array}{l}\text { Mostra a consistência } \\
\text { interna do construto }\end{array}$ & $>0,70$ & Kline (2011) \\
\hline Significância (p-value) & $\begin{array}{l}\text { Examina a relação entre } \\
\text { duas ou mais medidas }\end{array}$ & $<0,05$ & Byrne (2009) e Hair et al. (2009) \\
\hline
\end{tabular}

Fonte: Adaptado de Hair et al. (2009), Byrne (2009) e Kline (2011)

Por fim, procedeu-se à análise do modelo estrutural e análise de regressão para verificar o suporte estatístico das hipóteses de pesquisa.

\section{ANÁLISE E DISCUSSÃO DOS RESULTADOS}

Para verificar as características das empresas estudadas, foi realizada uma estatística descritiva com 0 porte e setor de atuação. No Gráfico 1, pode-se observar que boa parte das empresas (54\%) são grandes empresas, ao passo que os demais portes estão bem distribuídos na pesquisa. Essa diversidade reflete as características das empresas filiadas à FNQ e IPEG. A maior participação das grandes empresas pode significar um reflexo do perfil inovador das grandes e pequenas empresas, uma vez que as grandes empresas comumente possuem um processo de inovação mais estruturado (FRANCO; HAASE, 2010), o que pode ter influenciado a participação na pesquisa; e as pequenas empresas, processos de inovação mais informais (FRANCO; HAASE, 2010). Em relação ao setor de atuação, nota-se uma maior concentração em empresas de serviços (74\%), o que também evidencia uma característica das empresas filiadas à FNQ e IPEG e também representa uma tendência de distribuição das empresas no país. 
Gráfico 1: Modelo de Diagnóstico da Organização Inovadora

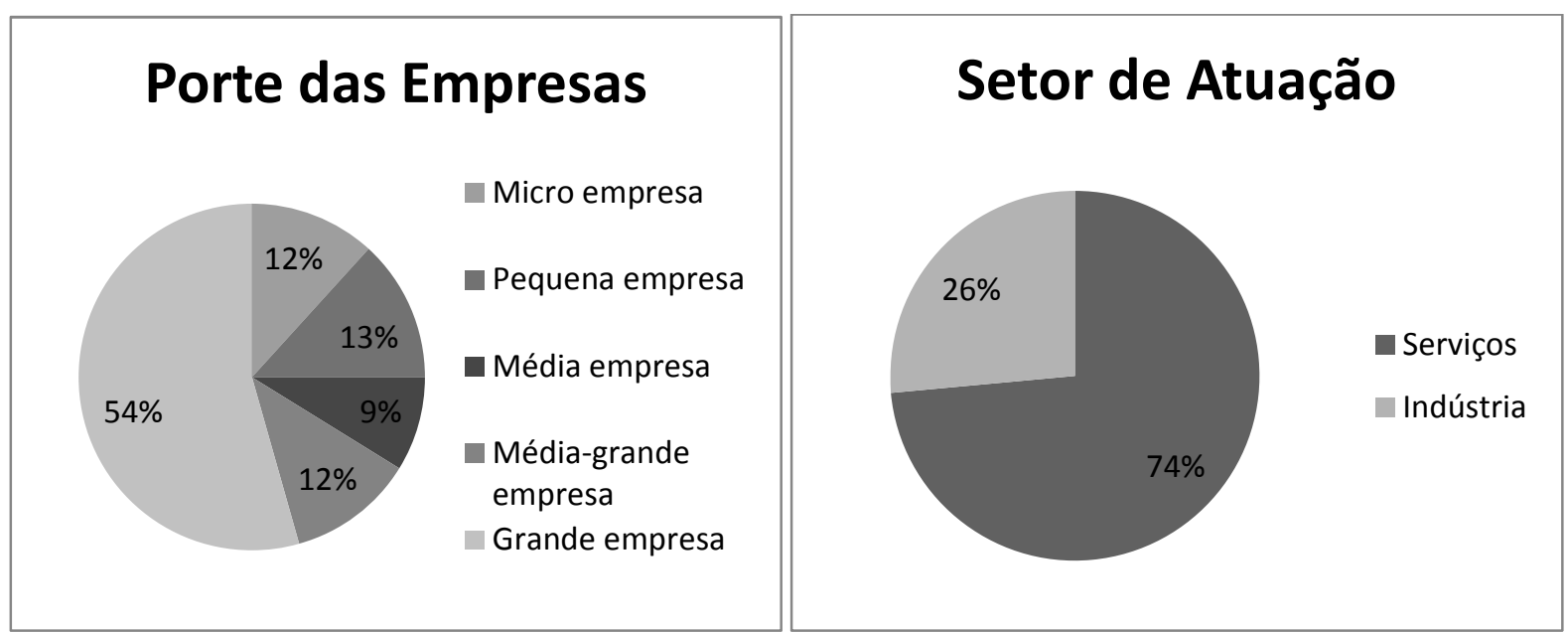

Fonte: Elaborado pelos autores (2015)

Para verificar a adequação dos fatores correspondentes a cada competência, foi rodada uma análise fatorial confirmatória, conforme disposto na Tabela 1.

Tabela 1: Indicadores de confiabilidade da AFC

\begin{tabular}{|c|c|c|c|c|c|c|c|}
\hline Variável & & $\begin{array}{l}\text { Construto de } \\
2^{\mathrm{a}} \text { ordem }\end{array}$ & $\begin{array}{c}\text { Carga } \\
\text { Padronizada }\end{array}$ & $\mathbf{R}^{2}$ & $\begin{array}{c}\text { Alfa de } \\
\text { Cronbach }\end{array}$ & AVE & $\begin{array}{c}\text { Confiabilidade } \\
\text { composta }\end{array}$ \\
\hline GESTÃO_1 & $<--$ & Liderança & 0,798 & 0,637 & \multirow{8}{*}{0,878} & \multirow{8}{*}{0,540} & \multirow{8}{*}{0,904} \\
\hline GESTÃO_2 & $<-$ & Liderança & 0,720 & 0,518 & & & \\
\hline GESTÃO_3 & $<--$ & Liderança & 0,740 & 0,548 & & & \\
\hline GESTÃO_4 & $<--$ & Liderança & 0,739 & 0,546 & & & \\
\hline LIDERANCA_1 & $<--$ & Liderança & 0,792 & 0,627 & & & \\
\hline \begin{tabular}{|l|} 
LIDERANCA_2 \\
\end{tabular} & $<-$ & Liderança & 0,706 & 0,498 & & & \\
\hline PSINAIS_1 & $<--$ & Liderança & 0,676 & 0,457 & & & \\
\hline PSINAIS_2 & $<--$ & Liderança & 0,701 & 0,491 & & & \\
\hline Al_1 & $<--$ & Meio Inovador & 0,615 & 0,378 & \multirow{7}{*}{0,838} & \multirow{7}{*}{0,504} & \multirow{7}{*}{0,875} \\
\hline ALIANÇA_1 & $<--$ & Meio Inovador & 0,554 & 0,307 & & & \\
\hline CONHECIMENTO_1 & $<--$ & Meio Inovador & 0,829 & 0,687 & & & \\
\hline CONHECIMENTO_2 & $<--$ & Meio Inovador & 0,850 & 0,723 & & & \\
\hline IE_1 & $<--$ & Meio Inovador & 0,676 & 0,457 & & & \\
\hline IE_2 & $<--$ & Meio Inovador & 0,723 & 0,523 & & & \\
\hline PC_1 & $<--$ & Meio Inovador & 0,675 & 0,456 & & & \\
\hline CULTURA_1 & $<--$ & Pessoas & 0,877 & 0,769 & \multirow{4}{*}{0,924} & \multirow{4}{*}{0,595} & \multirow{4}{*}{0,936} \\
\hline CULTURA_2 & $<--$ & Pessoas & 0,782 & 0,612 & & & \\
\hline CULTURA_3 & $<--$ & Pessoas & 0,682 & 0,465 & & & \\
\hline CULTURA_4 & $<-$ & Pessoas & 0,802 & 0,643 & & & \\
\hline
\end{tabular}

Revista Alcance - Eletrônica - vol. 23 - n. 3 - jul./set. 2016 


\begin{tabular}{|c|c|c|c|c|c|c|c|}
\hline MOTIVAÇÃO_1 & |<--- & Pessoas & 0,778 & 0,605 & & & \\
\hline MOTIVAÇÃO_2 & $<--$ & Pessoas & 0,801 & 0,642 & & & \\
\hline QVIDA_1 & $\mid<--$ & Pessoas & 0,712 & 0,507 & & & \\
\hline QVIDA_2 & <--- & Pessoas & 0,737 & 0,543 & & & \\
\hline QVIDA_3 & $\mid<--$ & Pessoas & 0,786 & 0,618 & & & \\
\hline QVIDA_4 & $<---$ & Pessoas & 0,741 & 0,549 & & & \\
\hline APRENDIZ_1 & \begin{tabular}{|l|}
$<---$ \\
\end{tabular} & Processos & 0,515 & 0,265 & \multirow{15}{*}{0,934} & \multirow{15}{*}{0,527} & \multirow{15}{*}{0,943} \\
\hline APRENDIZ_2 & $|<--|$ & Processos & 0,619 & 0,383 & & & \\
\hline CAPITAL_1 & $<--$ & Processos & 0,854 & 0,729 & & & \\
\hline CAPITAL_2 & <--- & Processos & 0,760 & 0,578 & & & \\
\hline IDEIAS_1 & \begin{tabular}{|l|}
$<---$ \\
\end{tabular} & Processos & 0,696 & 0,484 & & & \\
\hline IDEIAS_2 & \begin{tabular}{|l|}
$<---$ \\
\end{tabular} & Processos & 0,727 & 0,529 & & & \\
\hline IDEIAS_3 & \begin{tabular}{|l|}
$<---$ \\
\end{tabular} & Processos & 0,642 & 0,412 & & & \\
\hline PROCESSO_1 & \begin{tabular}{|l|}
$<---$ \\
\end{tabular} & Processos & 0,844 & 0,712 & & & \\
\hline PROCESSO_2 & $\mid<---$ & Processos & 0,842 & 0,709 & & & \\
\hline PROCESSO_3 & $\mid<---$ & Processos & 0,843 & 0,711 & & & \\
\hline \begin{tabular}{|l} 
PROJETOS_1 \\
\end{tabular} & <--- & Processos & 0,666 & 0,444 & & & \\
\hline PROJETOS_2 & $\mid<--$ & Processos & 0,817 & 0,667 & & & \\
\hline REDES_1 & $<---$ & Processos & 0,664 & 0,441 & & & \\
\hline REDES_2 & $\mid<--$ & Processos & 0,662 & 0,438 & & & \\
\hline REDES_3 & \begin{tabular}{|l|}
$<---$ \\
\end{tabular} & Processos & 0,639 & 0,408 & & & \\
\hline IMPACTO_1 & <--- & Resultado & 0,837 & 0,701 & \multirow{12}{*}{0,926} & \multirow{12}{*}{0,558} & \multirow{12}{*}{0,937} \\
\hline IMPACTO_2 & \begin{tabular}{|l|}
$<---$ \\
\end{tabular} & Resultado & 0,846 & 0,716 & & & \\
\hline IMPACTO_3 & $<---$ & Resultado & 0,785 & 0,616 & & & \\
\hline IMPLEMENT_1 & <--- & Resultado & 0,779 & 0,607 & & & \\
\hline OBJETIVOS_1 & $<---$ & Resultado & 0,774 & 0,599 & & & \\
\hline OBJETIVOS_2 & $<---$ & Resultado & 0,753 & 0,567 & & & \\
\hline RESULT_1 & $<---$ & Resultado & 0,624 & 0,389 & & & \\
\hline RESULT_2 & $<--$ & Resultado & 0,803 & 0,645 & & & \\
\hline RESULTPROC_1 & \begin{tabular}{|l|}
$<---$ \\
\end{tabular} & Resultado & 0,862 & 0,743 & & & \\
\hline RESULTPROC_2 & <--- & Resultado & 0,728 & 0,530 & & & \\
\hline RESULTPROC_3 & \begin{tabular}{|l|}
$<---$ \\
\end{tabular} & Resultado & 0,512 & 0,262 & & & \\
\hline RESULTPROC_4 & \begin{tabular}{|l|}
$<--$ \\
\end{tabular} & Resultado & 0,565 & 0,319 & & & \\
\hline
\end{tabular}

Fonte: Elaborada pelos autores (2015) 
Após ser rodada uma primeira análise fatorial confirmatória, houve a necessidade de exclusão de algumas variáveis, o que permitiu uma adequação do modelo utilizado aos propósitos desta pesquisa. Esta adequação está disposta na Tabela 2.

Tabela 2: Indicadores de confiabilidade da AFC ajustada

\begin{tabular}{|c|c|c|c|c|c|c|c|}
\hline Variável & $<-$ & $\begin{array}{l}\text { Construto de } \\
2^{\mathrm{a}} \text { ordem }\end{array}$ & $\begin{array}{c}\text { Carga } \\
\text { Padronizada }\end{array}$ & $\mathbf{R}^{2}$ & $\begin{array}{c}\text { Alfa de } \\
\text { Cronbach }\end{array}$ & AVE & $\begin{array}{l}\text { Confiabilidade } \\
\text { composta }\end{array}$ \\
\hline GESTÃO_1 & $<--$ & Liderança & 0,852 & 0,726 & \multirow{5}{*}{0,843} & \multirow{5}{*}{0,612} & \multirow{5}{*}{0,887} \\
\hline GESTÃO_2 & $<--$ & Liderança & 0,749 & 0,561 & & & \\
\hline GESTÃO_3 & $<--$ & Liderança & 0,741 & 0,549 & & & \\
\hline GESTÃO_4 & $<--$ & Liderança & 0,774 & 0,599 & & & \\
\hline LIDERANÇA_1 & $<--$ & Liderança & 0,790 & 0,624 & & & \\
\hline CONHECIMENTO_1 & $<--$ & Meio Inovador & 0,886 & 0,785 & \multirow{3}{*}{0,799} & \multirow{3}{*}{0,719} & \multirow{3}{*}{0,884} \\
\hline CONHECIMENTO_2 & $<--$ & Meio Inovador & 0,913 & 0,834 & & & \\
\hline IE_2 & $<--$ & Meio Inovador & 0,735 & 0,540 & & & \\
\hline CULTURA_1 & $<--$ & Pessoas & 0,885 & 0,783 & \multirow{8}{*}{0,917} & \multirow{8}{*}{0,634} & \multirow{8}{*}{0,933} \\
\hline CULTURA_2 & $<--$ & Pessoas & 0,750 & 0,563 & & & \\
\hline CULTURA_4 & $<--$ & Pessoas & 0,821 & 0,674 & & & \\
\hline MOTIVAÇÃO_1 & $<--$ & Pessoas & 0,795 & 0,632 & & & \\
\hline MOTIVAÇÃO_2 & $<--$ & Pessoas & 0,815 & 0,664 & & & \\
\hline QVIDA_2 & $<--$ & Pessoas & 0,733 & 0,537 & & & \\
\hline QVIDA_3 & $<--$ & Pessoas & 0,798 & 0,637 & & & \\
\hline QVIDA_4 & $<--$ & Pessoas & 0,765 & 0,585 & & & \\
\hline CAPITAL_1 & $<--$ & Processos & 0,901 & 0,812 & \multirow{7}{*}{0,939} & \multirow{7}{*}{0,737} & \multirow{7}{*}{0,951} \\
\hline CAPITAL_2 & $<--$ & Processos & 0,830 & 0,689 & & & \\
\hline IDEIAS_2 & $<--$ & Processos & 0,726 & 0,527 & & & \\
\hline PROCESSO_1 & $<--$ & Processos & 0,911 & 0,830 & & & \\
\hline PROCESSO_2 & $<--$ & Processos & 0,890 & 0,792 & & & \\
\hline PROCESSO_3 & $<--$ & Processos & 0,873 & 0,762 & & & \\
\hline PROJETOS_2 & $<--$ & Processos & 0,861 & 0,741 & & & \\
\hline IMPACTO_1 & $<--$ & Resultado & 0,795 & 0,632 & \multirow{6}{*}{0,903} & \multirow{6}{*}{0,673} & \multirow{6}{*}{0,925} \\
\hline IMPACTO_2 & $<--$ & Resultado & 0,850 & 0,723 & & & \\
\hline IMPACTO_3 & $<--$ & Resultado & 0,807 & 0,651 & & & \\
\hline IMPLEMENT_1 & $<--$ & Resultado & 0,820 & 0,672 & & & \\
\hline OBJETIVOS_1 & $<--$ & Resultado & 0,829 & 0,687 & & & \\
\hline OBJETIVOS_2 & $<--$ & Resultado & 0,818 & 0,669 & & & \\
\hline
\end{tabular}

Fonte: Elaborada pelos autores (2015) 
Após a análise fatorial exploratória, procedeu-se a uma regressão múltipla (Tabela 3), no intuito de verificar se há suporte estatístico para as hipóteses deste estudo.

Tabela 3: Regressão

\begin{tabular}{|c|c|c|c|c|c|}
\hline Coefficients & & & & & \\
\hline Term & Coef & SE Coef & T-Value & P-Value & VIF \\
\hline Constant & $-0,312$ & 0,43 & $-0,73$ & 0,47 & \\
\hline MÉDIA_MEIO & 0,166 & 0,143 & 1,16 & 0,25 & 3,24 \\
\hline MÉDIA_LIDERANCA & $-0,031$ & 0,214 & $-0,15$ & 0,884 & 5,5 \\
\hline MÉDIA_PESSOA & 0,275 & 0,169 & 1,63 & 0,107 & 4,24 \\
\hline MÉDIA_PROCESSO & 0,559 & 0,156 & 3,59 & 0,001 & 5,12 \\
\hline
\end{tabular}

Fonte: Elaborada pelos autores (2015)

Após isso, foi realizada a adequação da regressão (Tabela 4).

Tabela 4: Regressão ajustada

\begin{tabular}{|l|r|r|r|r|r|}
\hline Term & \multicolumn{1}{l|}{ loef } & \multicolumn{1}{l|}{ SE Coef } & T-Value & P-Value & \multicolumn{1}{l|}{ VIF } \\
\hline Constant & $-0,365$ & 0,328 & $-1,11$ & 0,268 & \\
\hline ZMED_LID & 0,026 & 0,145 & 0,18 & 0,859 & 3,74 \\
\hline ZMED_MEIO & 0,2416 & 0,0999 & 2,42 & 0,018 & 3,64 \\
\hline ZMED_PESS & 0,356 & 0,132 & 2,7 & 0,008 & 3,38 \\
\hline ZMED_PROC & 0,38 & 0,0909 & 4,18 & 0 & 3,64 \\
\hline
\end{tabular}

Fonte: Elaborada pelos autores (2015)

A regressão permitiu suporte estatístico para afirmar a influência positiva das competências meio inovador, pessoas e processos sobre o resultado. Para reforçar os achados, foi elaborada uma modelagem de equações estruturais, com as cargas e p-values dispostos nas Figuras 2 e 3, respectivamente. 
Figura 2: Modelagem de equações estruturais - carga

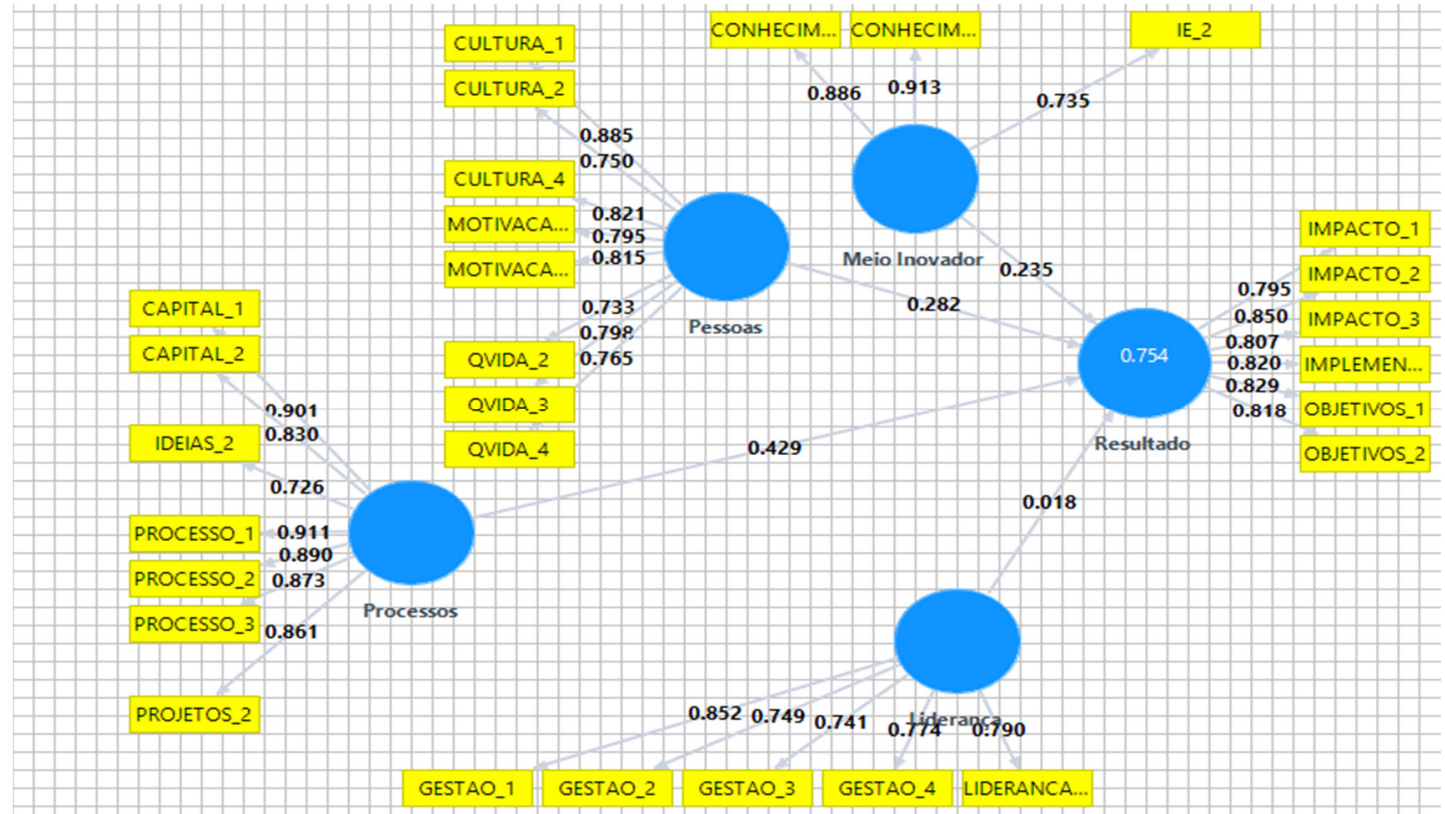

Fonte: Elaborada pelos autores (2015).

Figura 3: Modelagem de equações estruturais - p-values

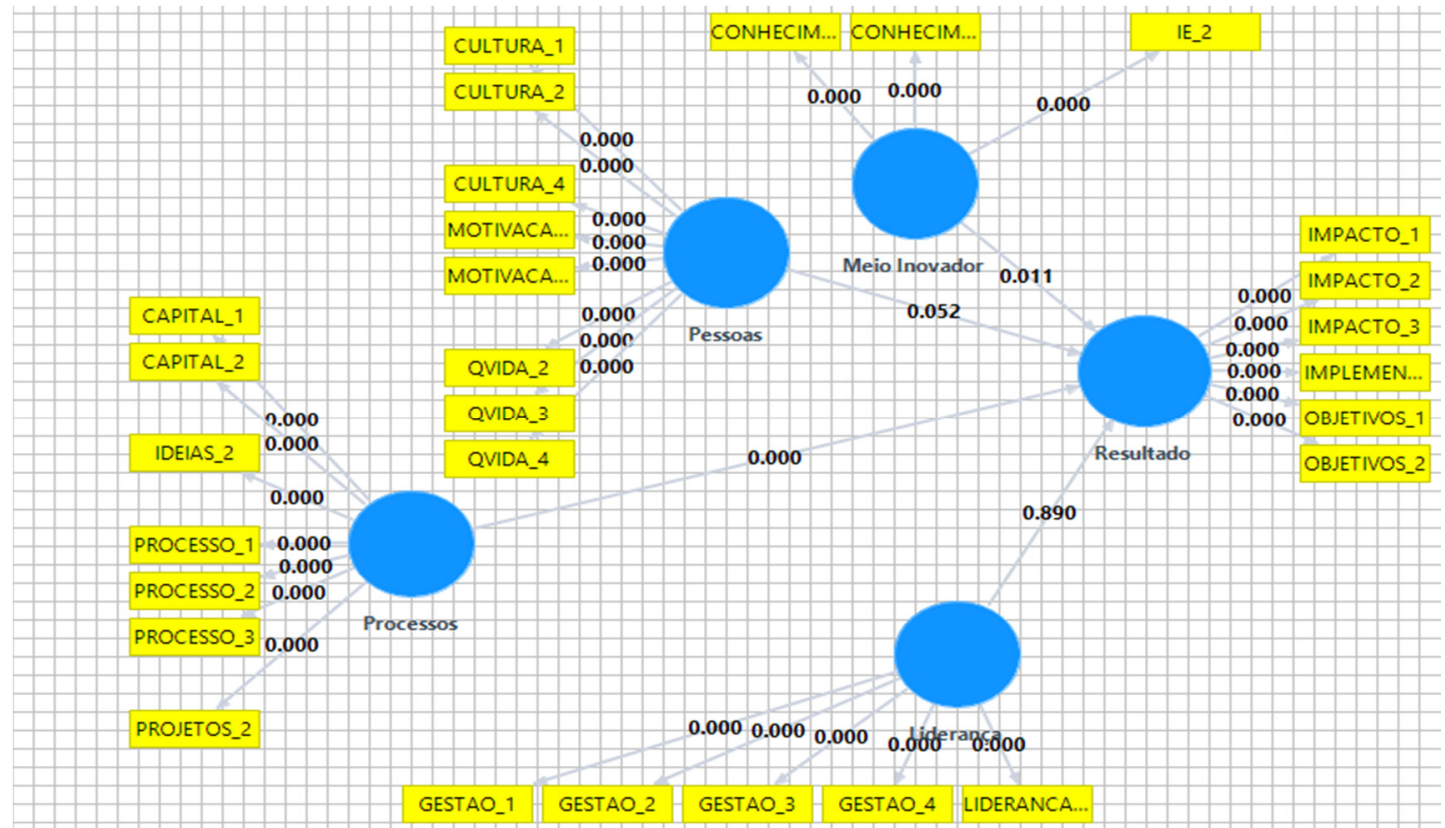

Fonte: Elaborada pelos autores (2015).

Tanto por meio da regressão como por modelagem de equações estruturais há suporte estatístico para afirmar a influência positiva do meio inovador, pessoas e processos sobre o resultado. Apenas a liderança não apareceu como determinante do resultado, uma vez que não houve suporte estatístico para esta inferência. Nesse sentido, as hipóteses $\mathrm{H} 2, \mathrm{H} 3$ e H4 foram suportadas e as hipóteses $\mathrm{H} 1$ e H5 não obtiveram suporte estatístico.

Embora a competência liderança e intenção estratégica não tenha obtido suporte estatístico, esta é responsável por impulsionar a forma como a organização enxerga e institui a inovação na organização (CROSSAN; APAYDIN, 2009; TANG, 1998), assim, a depender da forma como esta enxerga a inovação, é criado um meio inovador que impulsione ações de inovação. Estas ações são executadas pelas pessoas, que, a depender de sua motivação e da forma como enxergam a inovação como parte da cultura da organização, irão empreender 
as ações de inovação. Todos esses aspectos irão ocorrer mediante a criação de um processo de inovação, que interligue as competências anteriores com os resultados de inovação. Por fim, os resultados de inovação são frutos de vários fatores, mas refletem a capacidade da empresa em lidar com as competências anteriores. Este processo cíclico de competências faz com que a organização se diferencie no mercado, de modo que deficiências em todas as competências mostram falhas na capacidade de a empresa se comunicar com o mercado e, principalmente, inovar.

A falta de suporte estatístico para as hipóteses $\mathrm{H} 1$ e $\mathrm{H} 5$ podem significar uma importante lacuna ao indicar que a organização pode ser capaz de criar mecanismos de inovação que substituam a falta de definição dos rumos que a organização deve tomar e da criação de um ambiente favorável à inovação que venha de cima para baixo, responsabilidades da competência liderança e intenção estratégica. Entretanto, essa substituição pode funcionar em curto prazo ou em momentos de baixo nível de incerteza ambiental (CHESBROUGH, 2010). A inovação exige esforços contínuos e permanentes que, se não bem definidos, podem prejudicar os esforços de inovação (TIDD; BESSANT; PAVITT, 2008). A gestão da inovação deve, portanto, ser feita considerando todas as competências em conjunto e os efeitos que cada competência possui no resultado final de inovação (WEERAWARDENA; MAVONDO, 2011).

Tais resultados reforçam a necessidade de as empresas pensarem em inovação como fruto de ideia, ação e resultados e empreenderem esforços para maximizar sua capacidade inovadora em prol da perenidade do negócio. Uma empresa incapaz de inovar ou que não consiga acompanhar as atuais demandas de mercado pode perder rapidamente sua posição competitiva e ser substituída por outras que melhor compreendam a dinâmica de mercado.

\section{Considerações Finais, Limitações e Recomendações}

O artigo teve como objetivo identificar lacunas na gestão de inovação de empresas do Instituto Paulista de Excelência da Gestão e da Fundação Nacional da Qualidade com base na metodologia do Fórum de Inovação da FGVIEAESP, que tem como base cinco competências da organização inovadora: liderança e intenção estratégica; meio inovador interno; pessoas; processos de inovação; e resultados. 0 método de pesquisa adotado foi a survey.

Os resultados da aplicação do modelo revelam uma influência positiva das competências meio inovador interno, pessoas e processos de inovação sob a competência resultado. Isso ocorre porque a gestão da inovação exige 0 envolvimento da organização em aspectos que conectem a empresa aos seus objetivos de inovação. Assim, as organizações devem se concentrar em criar mecanismos que potencializem as competências anteriores em um sistema dinâmico de inovação, representado por um processo de inovação forte e que potencialize as competências anteriores. Adicionalmente, as empresas devem cuidar para que suas competências mantenham sinergia entre si, evitando, assim, um processo de inovação que se mostre ineficaz no longo prazo e que, portanto, não gere resultado. Apesar da competência liderança e intenção estratégia não ter se mostrado estatisticamente positiva, cabe ressaltar que esta é responsável pela forma como a organização enxerga e institui a inovação na organização, sendo, portanto, importante sua articulação com as demais competências para bons resultados de inovação.

Como limitações desta pesquisa, o estudo reflete um conjunto de empresas com características diferentes, o que pode ter influenciado nos resultados obtidos quanto às hipóteses $\mathrm{H} 1 \mathrm{e} \mathrm{H}$. Para futuras pesquisas, sugere-se a realização de estudos com base no modelo de diagnóstico da organização inovadora proposto pelo Fórum de Inovação FGV/EAESP (2014) e a realização de um estudo de caso que possa explorar em profundidade como estas competências se relacionam nas organizações.

Como contribuição prática, o artigo chama a atenção para a dificuldade de as organizações gerarem resultado de inovação e o coloca como consequência de competências que 0 antecedem. Tal insight permite às empresas compreenderem a necessidade de interligarem as competências de inovação a um processo dinâmico e interativo, que permita gerar resultados em longo prazo. Como contribuição teórica, o artigo traz como proposta o modelo de diagnóstico da organização inovadora proposto pelo Fórum de Inovação FGV/EAESP (2014). Cabe destacar que poucos são os modelos que se propõem a articular em um modelo único um conjunto de competências que abarque empresas com diferentes características, sendo, portanto, uma importante contribuição desta pesquisa. 


\section{REFERÊNCIAS}

ALSAATY, F. M. A model for building innovation capabilities in small entrepreneurial firms. Academy of Entrepreneurship Journal, vol. 17, n. 1, 2011.

ÁLVARES, A. C. T. The 3M way to innovation: balancing people and profit. Revista de Administração de Empresas, vol. 41, n. 3, 2001.

BABBIE, E. Métodos de pesquisas de survey. Belo Horizonte: Editora UFMG, 2001.

BAO, Y.; CHEN, X.; ZHOU, K. Z. External learning, market dynamics, and radical innovation: Evidence from China's high-tech firms. Journal of Business Research, vol. 65, pp. 1226-1233, 2012.

BARBIERI, J. C. Organizações inovadoras: estudos e casos brasileiros. RJ: Editora da FGV, 2003.

BARBIERI, J. C.; ÁLVARES, A. C. T. Meio inovador empresarial: conceitos, modelos e casos. Revista IMES Administração, vol. 56, set./dez, 2002.

BENNIS, W. G.; NANUS, B.; SIMÕÉS, A. B. Líderes: estratégias para assumir a verdadeira liderança. Editorial Harbra, 1988.

BORCHARDT, P.; SANTOS, G. V. Gestão de ideias para inovação: transformando a criatividade em soluções práticas. Revista de Administração e Inovação, vol. 11, n. 1, pp. 203-237, 2014.

BYRNE, B. M. Structural equation modeling with AMOS: basic concepts, applications, and programming. 2. ed. New York: Taylor \& Francis Group, 2009.

CARAYANNIS, E.; GRIGOROUDIS, E. Linking innovation, productivity, and competitiveness: implications for policy and practice. The Journal of Technology Transfer, vol. 39, n. 2, pp. 199-218, 2014.

CARAYANNIS, E. G.; SAMARA, E. T.; BAKOUROS, Y. L. Innovation and Competitiveness: Case Study. In Innovation and Entrepreneurship. Springer International Publishing, pp. 47-72, 2015.

CASTELLS, M.; HALL, P. Las Tecnopolis del mundo: la formación de los complexos industriales del siglo XXI. Madrid: Alianza Editorial, 1994.

CHESBROUGH, H. Business model innovation: opportunities and barriers. Long Range Planning, vol. 43, pp. 354-363, 2010.

CHESBROUGH, H. Inovação aberta: como criar e lucrar com a tecnologia. trad. Luiz Claudio de Queiroz Faria; rev. téc. Jonas Cardona Venturini. Porto Alegre: Bookman, 2012.

CHRISTENSEN, C. M. The innovator's dilema. Harvard Business School Press. Harper Business Essencials, 1997.

CHRISTENSEN, C. M.; OVERDORF, M. Meeting the challenge of disruptive change. Harvard business review, vol. 78, n. 2, pp. 66-77, 2000.

CROSSAN, M. M.; APAYDIN, M. A multi-dimensional framework of organizational innovation: A systematic review of the literature. Journal of management studies, vol. 47, n. 6, pp. 1154-1191, 2009.

FRANCO, M.; HAASE, H. Failure factors in small and medium-sized enterprises: qualitative study from an attributional perspective. Int Entrep Manag J, vol. 6, pp. 503-521, 2010.

FÓRUM DE INOVAÇÃO FGVIEAESP. Modelo de diagnóstico da organização inovadora. RJ: Editora da FGV, 2014.

FLETCHER, M.; HARRIS, S. Knowledge acquisition for the internationalization of the smaller firm: content and sources. International Business Review, vol. 21, pp. 631-647, 2012.

HAIR, J. F.; BLACK, W. C.; BABIN, B. J.; ANDERSON, R. E.; TATHAM, R. L. Análise multivariada de dados. 6. ed. Porto Alegre: Bookman, 2009.

HAMEL, G.; PRAHALAD, C. K. Competing for the Future. Harvard Business School Press, Boston, 1994.

HARGADON, A.; SUTTON, R. I. Engineering the best business: building an innovation factory. IEEE Engineering Management Review, vol. 28, n. 4, pp. 17-23, 2000. 
HEGER, T.; ROHRBECK, R. Strategic foresight for collaborative exploration of new business fields. Technological Forecasting \& Social Change, vol. 79, pp. 819-831, 2012.

HURLEY, R. F.; HULT, G. T. M. Innovation, market orientation, and organizational learning: an integration and empirical examination. The Journal of Marketing, pp. 42-54, 1998.

JOHNSON, M. W.; CHRISTENSEN, C. M.; KAGERMANN, H. Reinventing your business model. Harvard business review, vol. 86, n. 12, pp. 57-68, 2008.

KLINE, R. B. Principles and practice of structural equation modeling. 3. ed. New York-NY: The Guilford Press, 2011.

LAWSON, B.; SAMSON, D. Developing innovation capability in organisations: a dynamic capabilities approach. International journal of innovation management, vol. 5, n. 3, pp. 377-400, 2001.

MACHADO, D. D. P. N. Organizações inovadoras: estudo dos fatores que formam um ambiente inovador. Revista de Administração e Inovação, vol. 4, n. 2, pp. 5-28, 2007.

MARKIDES, C. Strategic innovation. Sloan Management Review, vol. 38, pp. 9-24, 1997.

MONTEIRO, J. J. B. A. Inovação sistemática aplicada a sistemas industriais de polimerização. Revista de Administração e Inovação, vol. 12, n. 2, pp. 200-235, 2015.

OLIVEIRA, S. M.; ALVES, J. L. Influência das práticas de inovação aberta na prospecção de conhecimentos para a criação de valor em ambientes de alta complexidade sob condições de incerteza e imprevisibilidade. Revista de Administração e Inovação, vol. 11, n. 1, pp. 295-318, 2014.

PONOMARIOV, B.; TOIVANEN, H. Knowledge flows and bases in emerging economy innovation systems: Brazilian research 2005-2009. Research Policy, vol. 43, n. 3, pp. 588-596, 2014.

QUANDT, C. O.; SILVA, H. D. F. N.; FERRARESI, A. A.; FREGA, J. R. Programas de gestão de ideias e inovação: as práticas das grandes empresas na região sul do Brasil. Revista de Administração e Inovação, vol. 11, n. 3 , pp. 176-199, 2014.

RAMOS, A.; ZILBER, S. N. O impacto do investimento na capacidade inovadora da empresa. Revista de Administração e Inovação, vol. 12, n. 1, 2015.

ROBERTSON, P. L.; CASALI, G. L.; JACOBSON, D. Managing open incremental process innovation: absorptive capacity and distributed learning. Research Policy, vol. 41, pp. 822-832, 2012.

SALUNKE, S.; WEERAWARDENA, J.; MCCOLL-KENNEDY, J. R. Towards a model of dynamic capabilities in innovation-based competitive strategy: insights from project-oriented service firms. Industrial Marketing Management, vol. 40, pp. 1251-1263, 2011.

SAUNDERS, M.; LEWIS, P.; THORNILL, A. Research Methods for Business Students. 2. ed. Harlow, England: Pearson Education, 2000.

SILVA, S. E.; REIS, L. P. O processo de estruturação de recursos no contexto de uma empresa de base tecnológica de origem acadêmica (EBTA). Revista de Administração e Inovação, vol. 12, n. 2, pp. 153-179, 2015.

SMITH, M.; BUSI, M.; BALL, P.; VAN DER MEER, R. Factors influencing an organisation's ability to manage innovation: a structured literature review and conceptual model. International Journal of innovation management, vol. 12, n. 4, pp. 655-676, 2008.

SPISAK, B. R.; O'BRIEN, M. J.; NICHOLSON, N.; VAN VUGT, M. Niche Construction and the Evolution of Leadership. Academy of Management Review, vol. 40, n. 2, pp. 291-306, 2015.

TANG, H. K. An Integrative model of innovation in Organizations. Technovation, vol. 18, n. 5, pp. 297-309, 1998.

TIDD, J.; BESSANT, J.; PAVITT, K. Gestão da inovação. 3. ed. Porto Alegre: Bookman, 2008.

VASCONCELLOS, M. A. Excelência e Humanização da Produção. Monografia para acesso ao nível de professor titular. SP: FGV-EAESP, 1997.

WEERAWARDENA, J.; MAVONDO, F. T. Capabilities, innovation and competitive advantage. Industrial Marketing Management, vol. 40, pp. 1220-1223, 2011. 
ZACHER, H.; ROSING, K. Ambidextrous leadership and team innovation. Leadership \& Organization Development Journal, vol. 36, n. 1, pp. 54-68, 2015. 